Aus der chirurgischen Abteilung des Freimaurer-Krankenhauses zu Hamburg. (Oberarzt: Dr. H. Grisson.)

\title{
Giebt es eine primäre Invagination des Wurmfort- satzes in das Coecum?
}

Von Dr. Siegmund Pollag, Assistenzarzt.

(Mit I Abbildung.)

Die Klärung dieser alten Streitfrage dürfte durch die Bekanntgabe der folgenden Beobachtung herbeigeführt werden.

Der gjährige Knabe F. M. wurde schon im Februar 1915 wegen „Blinddarmentzündung" behandelt und im März desselben Jahres in einem andern hiesigen Krankenhause wegen „Nabelbruches" operiert. In Anschluß daran überstand er einen Scharlach. Am 27. VI. plötzlich Schmerzen im rechten Unterbauch, die genau den gleichen Charakter haben sollen, wie die im Februar verspürten. Kein Erbrechen; am Tage vorher und heute Stuhlgang. - Die Untersuchung ergibt bei normaler Temperatur einen etwas aufgetriebenen Leib mit ausgesprochener Druckempfindlichkeit und Bauchdeckenspannung in der Ileocökalgegend. Der Puls ist etwas frequent. Untersuchung der Organe und Urin o. B. - Da das ganze Krankheitsbild den Eindruck einer Appendicitis im Intermediärstadium macht, wird erst operiert, als eine Verschlimmerung des Allgemeinzustandes dieses Vorgehen indiciert erscheinen ließ.

Bei der am 29. VI. vorgenommenen Operation finden sich zahlreiche Verklebungen und Verwachsungen an der Ileocökalklappe. Der Wurmfortsatz findet sich erst nach längerem Suchen als adhärentes und etwas nach oben geschlagenes Organ, dessen auffallende Kürze sich dadurch erklärt, daß der proximale Teil ins Coecum hineinverlagert ist, während das distale Ende gleichsam wie ein Zapfen aus einem in der Dickdarmwand liegenden Grübchen in das Abdomen hineinhängt. Da ein Vorziehen des verlagerten Stückes zur Abtragung unmöglich ist, wird das das Invaginatum umgebende Stück Coecum mitexcidiert, das letztere durch mehrschichtige Naht vereinigt und die Bauchhöhle geschlossen. - Das stark hyperämische Invaginatum war zapfenartig in das Lumen des Coecum vorgetrieben und zeigte deutlich mehrere Schleimhautfalten; da, wo das ins Abdomen ragende di- 
stale Stïck begann, war eine deutliche Stenose vorhanden. Am Coecum oder Ileum werden keine Zeichen von gewesener oder bestehender Intussuszeption wahrgenommen. Die bestehenden Verhältnisse werden sehr klar durch die folgende photographische Skizze erläutert.

Nachdem der Heilungsverlauf durch einen gewissen Peritonismus einige Tage kompliziert gewesen war, erfolgte langsam Heilung und am 25. Tage nach der Operation Entlassung in beschwerdefreiem Zustande.

Die Katamnese des Falles läßt erkennen, daß mehrere für das Krankheitsbild der Invagination des Processus vermiformis

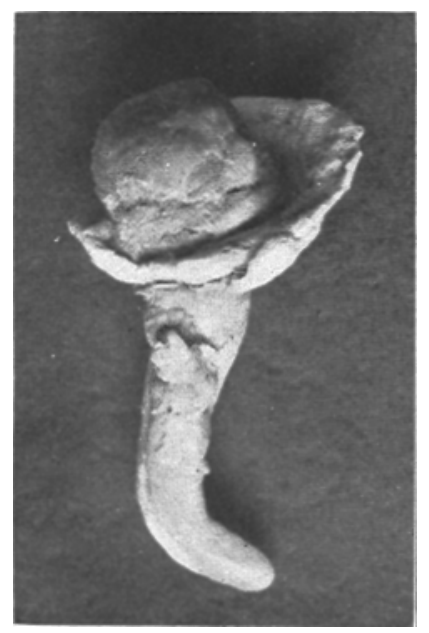

Fig. I.

typische Tatsachen auch hier zu konstatieren waren. Einmal das jugendliche Alter. Es ist nur eine Invagination des Wurmfortsatzes bei Ileocökal-Intussuszeption bei einer 42 jährigen Frau von $\mathrm{H}$ a a s le $\mathrm{r}$ und kürzlich die Entzündung eines Wurmfortsatzes bei bestehender chronischer ileocökaler Invagination bei einem $2 \mathrm{I}$ jährigen Unteroffizier von Le d d e r h o s e beschrieben worden; sonst handelte es sich bei allen bekannt gewordenen Fällen, sofern nicht, wie bei J a la g u i ers Fall I, die Altersangabe fehlt, stets um Kinder jeden Alters. Zweitens ergibt die Anamnese, daß es sich um eine chronische Erkrankung handelte, wie es die Regel zu sein pflegt, und als drittes Moment erweist sich eine 
große Übereinstimmung im Bilde der klinischen Symptome mit denen der Appendicitis acuta.

Was aber in diesem Falle völlig neu war, ist das isolierte Bestehen einer Invagination des Processus vermiformis ohne Lageverschiebung des Cöcum oder lleum. In der ganzen, sehr ausgedehnten Literatur, die mir zur Verfügung stand, konnte ich nur bei J a 1 a g u i ers Fall 2 nicht sicher entscheiden, ob dieses Vorkommnis nicht damals schon einmal vorgelegen hat; auf alle Fälle weist der Autor mit keinem Worte darauf als etwas Besonderes hin. Unser Operationspräparat war bis zu einer Stelle invaginiert, wo eine frühere Attacke die Appendix strikturiert hatte, was wiederum einer alten Erfahrungstatsache entspricht. Der über den Fall konsultierte Pathologe äußerte sich, daß er diese Beobachtung noch nie gemacht habe, und verwies auf S. 820 des II. Bandes des Aschoffschen Lehrbuches der pathologischen Anatomie, wo folgender Passus steht: „Noch seltener sind aufsteigende Invaginationen. Sehr merkwürdig ist der wiederholt gemachte Befund eines umgestülpten Wurmfortsatzes bei Invaginatio ileocoecalis. Diese Umstülpung ist, wie ich mit W il m s annehme, stets eine sekundäre."

W il ms widmet in seinem, ,Ileus" dieser Frage eine längere Betrachtung. Er kommt zu dem Schlusse, daß erst nach vollzogener Invagination des Darmes durch die in demselben als Folge der mit Ödem der Spitze beginnenden Stauung auftretende Spannung und Dehnung eine Umstülpung des Processus vermiformis ermöglicht und vielleicht noch durch die peristaltische Tätigkeit der äußeren Scheide erleichtert wird. Allerdings genügt auch schon die Spannung allein, um eine handschuhfingerartige Ausstülpung zu verursachen. Mit dieser Auslegung stellt er sich ausdrücklich in Gegensatz zu einer großen Anzahl Autoren, vor allem Ackermann, der auf Grund einer eingehenden Żusammenstellung der Literatur zum Schlusse kommt, daß die Invagination des Processus vermiformis das Primäre darstelle.

Ackermann unterscheidet komplette und inkomplette Einstülpungen, zu denen ein normaler Wurmfortsatz nicht geeignet erscheint, während eine insuffiziente Gerlachsche Klappe oder alte appendicitische Veränderungen, ev. sogar gewaltsame Entleerungen der Appendix dazu prädisponieren. $\mathrm{Zu}$ letzteren 
Fällen würden dann alle diejenigen gehören, wo Invagination bis $z u$ einer stenotischen Stelle erfolgt, und um eine solche an der Striktur endende Invagination hat es sich auch bei unserem Kranken gehandelt. Der Vorgang wäre dann nach Acker$m$ a n $n$ ähnlich zu denken wie beim Divertikel, indem ein fixiertes Darmstück sich, entsprechend den beim Anus praeternaturalis vorliegenden Verhältnissen, gegen eine Öffnung vordrängt. Wilms würde diesen Modus anerkennen, wenn es sich stets um divertikelartige und nicht um so enge Organe, wie der doch meist normale Wurmfortsatz, gehandelt hätte, die sich nicht durch eigene Peristaltik, sondern nur durch die oben beschriebenen, äußern Kräfte umzustülpen vermögen, zumindest in ihren distalen, doch sicher nicht erweiterten Partien. Damit erklärt Wilms auch die Tatsache, daß eine Stenose die weitere Einstülpung verhindert. Als Hauptstützpunkt seiner Beweisführung führt $\mathrm{W}$ il ms die Frage an, warum eine als möglich postulierte primäre Einstülpung des Wurmfortsatzes denn noch nie ohne gleichzeitige Invagination des Cöcums gefunden worden sei und sagt: „Wir kennen keinen einzigen Fall, in welchem ein Wurmfortsatz eingestuilpt im Cöcum sich gefunden hätte." Wenn auch der eben zitierte Satz die Fragc offen läßt, ob Wilms bei dieser Forderung einer seine Ansicht widerlegenden Beobachtung nur eine komplette Einstülpung als beweisführend ansehen würde, so muß ich doch daran festhalten, daß unser Fall die sichere Entscheidung der Titelfrage darstellt; denn es hat sich dabei um eine irreponible, wenn auch inkomplette, aber sicher isoliert ohne gleichzeitig vorhandene Invagination von Darmteilen bestehende Einstülpung des Wurmfortsatzes gehandelt. Denn, daß eine chronische Darmintussuszeption, vielleicht intermittierender Art, bestanden hätte, und am Operationstage gelöst gewesen wäre, ist, wenn auch theoretisch denkbar, doch höchst unwahrscheinlich, vor allem auch mit Rücksicht auf den erreichten Erfolg. Bei der Operation im März I9I5 hatte es sich nach der derzeitigen Krankengeschichte nur um einen radikal operierten Nabelbruch gehandelt, während die seit Januar bestehenden, mit Erbrechen vergesellschafteten Leibschmerzen darauf hinweisen, daß die bei uns beobachtete Affektion schon zu jener Zeit bestanden haben muß, also chronisch geworden war. 
Die Literatur über Intussuszeption ist unermeßlich und auch der uns interessierenden Spezialfrage der Einstülpung des Wurmfortsatzes sind erstaunlich viele Arbeiten, besonders englischer Autoren, gewidmet. Es sollen auch bloß die auf die $\Lambda$ ppendix bezüglichen Darstellungen bei dem im folgenden in Kürze niedergelegten Exkurs über das uns beschäftigende Krankheitsbild und seine Erklärung, wie sie sich mir nach dem Studium aiieser Literatur ergeben hat, berücksichtigt werden.

Unter kolikähnlichen, meist paroxysmenartig auftretenden Schmerzen, die bald diffuser verbreitet sind, bald typisch in die Ileocöcalgegend lokalisiert werden, erkranken die Patienten. Meist tritt heftiges Erbrechen ein, in vielen Fällen auch mehr oder weniger profuse Diarrhöen, die schleimigen, gelegentlich blutigen Charakter haben. In seltenen Fällen sieht man Obstipation und, was bei bestehender Intussuszeption nicht wundernehmen kann, Stuhl- und Windverhaltung. Auch Fiebersteigerungen sind beobachtet; das Allgemeinbefinden ist stark alteriert, der Puls frequent, die Atmung meist beschleunigt. Man findet im rechten Unterbauch eine bald verbreitert, bald zirkumskript am Mac Burney lokalisierte Druckempfindlichkeit, eine gewisse Bauchdeckenspannung und meist deutlich einen mehr oder weniger großen Tumor, der unter Umständen leicht verschieblich und schmerzhaft ist. Dieser letztere kann an verschiedenen Stellen auftreten und zeitweise ganz verschwinden, um plötzlich wieder zu erscheinen und den Symptomenkomplex einer akuten Appendicitis, die einer chronischen Affektion aufgepfropft ist, auszulösen. Die Größe des Tumors wechselt in den einzelnen Anfällen oft stark, die Intervalle pflegen völlig beschwerdefrei zu sein. So bestehen oft monatelang Darmstörungen ohne eigentliche Verschlußzeichen, und erst die Operation oder Autopsie ergibt dann eine Intussuszeption, bei der manchmal die Appendix entweder heftig miterkrankt (wie im Fall Ledderhose) oder mitinvaginiert ist. $\mathrm{Da}$ aber die Appendix mit ihrer bekannten starken Einwirkung auf das Befinden und ihren auffallenden Darmsymptomen erst den akuten Prozeß auslöst und damit das Rad der aktiven Therapie so eigentlich ins Rollen bringt, scheint mir sicher zu sein; daß eine isolierte Invagination der Appendix vorkommt und ohne Darmeinschiebung so heftige Erscheinungen 
macht, beweist unser Fall. Gelegentlich sind auch tuberkulöse Prozesse als ätiologisches Moment festgestellt worden (Pitts, Wright und Kowles Renshaw). Perforationen und Peritonitis treten auf, wenn Spontanreposition nicht eintritt oder die definitive Verwachsung der einscheidenden Teile nicht abgewartet wird und das Invaginatum sich vorzeitig abstößt.

Die eigentliche Invaginatio ileocoecalis ist relativ leicht $\mathrm{zu}$ diagnostizieren; sie kommt gelegentlich mit weiteren Darmeinscheidungen gemeinsam vor (Fall Riedel); eine Analuntersuchung sollte nie unterlassen werden. $\mathrm{Ob}$ aber die Appendix völlig unbeteiligt, ob sie miteingestülpt, ob sie in situ mit hineingezogen oder ob sie entzündet ist (wobei wir wiederum eine Entzündung der in situ mitinvaginierten und der ihrerseits eingestülpten Appendix unterscheiden müssen), wird bei dem Ineinanderfließen der subjektiven und objektiven Krankheitsbilder kaum je vorauszusagen sein. Daß die differentialdiagnostische Erwägung an den Praktiker herantreten kann, erwähnt $\mathrm{S}$ p rengel in seiner ,Appendicitis" (Deutsche Chirurgie, S. 453 und 483), ohne auf genauere Details einzugehen. Daß nach meiner Auffassung bei der anerkannten Chronizität solcher Fälle von ileocökaler Jnvagination sehr oft erst akute Komplikationen von Seite des mitergriffenen Wurmfortsatzes das Bild schwer erscheinen lassen und ein rasches Eingreifen erfordern, habe ich schon weiter oben angedeutet; desgleichen die ganz auffallende Bevorzugung des Kindesalters.

Die Therapie fällt zum Teil mit der der Invagination im allgemeinen zusammen. Da es sich um Kinder handelt, sind Insufflation und Eingießung vor der Operation stets zu versuchen; dabei würde sich wohl auch eine Mitinvagination der Appendix lösen oder aber durch ihr Zurückbleiben die bekannten Symptome hervorrufen. Wenn also unter den eben genannten Manipulationen der Tumor verschwindet, die appendicitischen Erscheinungen aber bleiben, kann man ruhig die Diagnose „Invagination des Processus vermiformis" stellen und operieren. Palliativoperationen, wie Anus praeternaturalis oder Darmausschaltung durch Enteroanastomose kommen dabei wohl nicht in Betracht, es sei denn, der Tumor sei sehr groß und stark in unlösliche Verwachsungen eingebettet; dagegen wird die Desinvagination mit nachheriger Fixation des Darmes, bzw. die Re- 
sektion (ev. sowohl des Intussuscipiens als auch des Intussusceptums) als Radikaloperation in Frage kommen. Gelingt die Reposition der Därme, wie es bei Kindern meist der Fall ist, ohne Vorlagerung derselben, so wird nach dem Vorgang von $\mathrm{R}$ a y das Mesenteriolum durch eine Naht an das Mesenterium befestigt und so dic Appendix am Zurückschlüpfen verhindert. Das ist nach meiner Auffassung von der Invagination der Appendix als dem primären Vorgang theoretisch zwar ein kausale Therapie, die Rezidive verhindern kann, in praxi aber soll man überhaupt die Appendix entfernen, was nach dem heutigen Stande unserer Ansichten gerechtfertigt erscheint, wenn man sie bei Operationen schon in der Hand hat; das um so mehr, als sie meistens bereits krankhafte Veränderungen zeigen wird, die in letzter Instanz wohl die Invagination verursacht haben, bzw. weil die Tatsache der Invagination als solcher allein schon eine genügende Indikation für clieses Vorgehen darstellt.

Wir haben bereits gesehen, wie Wilms und A s ch of f sich mit ihrer Auffassung, daß die Invagination des Processus vermiformis stets sekundär sei, in Gegensatz zu den meisten Autoren stellen, von denen besonders nachdrücklich J a la g u i e r, A ckermann, Haasler, Wright und Knowles Renshaw, d'A rcy Power und Ledderhose den Standpunkt vertreten, daß dic Affektion des Wurmfortsatzes die primäre sei. Wie W il m s sich den Vorgang im Gegensatz zu A ck e r man n denkt, ist bereits eingehend dargestellt worden. Wright und Know les Renshaw sahen die Appendix mit ihrer Wurzel in entzündlichem Zustande in das Cöcum eingestülpt, während das distale Ende wie ein „Pilzstengel“ darunter saß und in die Bauchhöhle ragte. Durch die Schwellung kam es dann erst zur eigentlichen Intussuszeption. Pitts erklärte die nach seiner Ansicht primäre Invagination der Appendix mit einer Polypenwirkung, wie wir sie bei anderen Organen auch kennen, um eine Darminvagination entstehen zu lassen. Ganz besonders wichtig für die Entscheidung der Frage erscheinen mir die Beobachtungen von Jalaguier, der zweimal Gelegenheit hatte, bei Hernienoperationen die Appendix im Scrotalsack zu finden. Nahm er sie zwischen die Finger, so vergrößerte sie sich zwei- bis dreifach, krümmte sich und wurde steif und diese Kontraktionen setzten 
sich auf das Cöcum fort. Bleibt also das Cöcum weich, so wird es bei Steifung im Wurmfortsatz eingedrückt und ist es einmal eingedrückt, dann steht der Weg zu großen ileocökalen Invaginationen offen. Geradezu den Wert einer experimentellen Studie hat die Operation eines 8 jährigen Knaben durch denselben Autor. Es fand sich eine Cökalgeschwulst, die aus einer Invagination eines Teiles des Wurmfortsatzes und der Valvula Bauhini bestand, welche etwa $2 \mathrm{~cm}$ engestülpt waren. Beim Ergreifen des Wurmfortsatzes und Eindrücken gegen das Cöcum stellte sich spontan eine Invagination bis ins Colon tranversum her. Ich muß mich darum denjenigen Autoren anschließen, die die Invagination der Appendix als das Primäre ansehen, um so mehr als ich das an sich schon sehr beweiskräftige Material dadurch noch endgültig stützen kann, daß ich den vorliegenden Fall von isolierter Invagination des Processus vermiformis bekannt gegeben habe.

Meinem verehrten Chef, Herrn Dr. Griss on, möchte ich an dieser Stelle für die Überlassung des Falles und die Anregung $z u$ seiner wissenschaftlichen Verwertung meinen verbindlichsten Dank aussprechen.

\section{Literaturverzeichnis.}

I. A ckermann, Uber die Umstülpung des Processus verniformis und ibre Beziehung zur Intussusception. v. Bruns' Beiträge zur klinischen Chirurgie I903, Bd. 37. S. 579

2. Alsberg. Darminvagination. Enzyklopädie Kocher-de Quervain Igor.

3. d'Arcy Power, a) Ileocoecal intussusception anots treatment. Edinburgh medical Journal 1897 June;

b) Hunterian lectures on the pathology and surgery of intussusception. British medical Journal I897. 13., 20., 27. Februar;

c) Case of ileo-iliac intussusception spontaneously cured. British medical Journal 1903. 25. April.

4. As ch off. Lehrbuch der Pathologie. Bd. 2, S. 820.

5. Braun, Invaginatio ileocoecalis. Berichte der medizinischen Gesellschaft zu Leipzig. Juli i 897 .

6. Club be, A clinical lecture on the diagnosis and treatment of intussusception. British medical Journal I90I. March 23.

7. Connor, Intussusception of the vermiform appendix. The Lancet rgo3. 29. August.

8. Dartigues, Invagination intestinale ascendante du caecum avec son 
appendice, du côlon et d'une partie du transverse. Laparotomie. Bulletin de la société anatomique de Paris 1897, No. 18.

9. Elgart, 2 operativ geheilte Fälle von Invaginatio ileocoecalis bei Erwachsenen. Wiener klin. Wochenschr. 1903, Nr. 32.

Io. Enderlen. Ärtlicher Verein zu Nürnberg. Sitzung vom I6. November I899. Münchn. med. Wochenschr. I900. 17. Juli, S. I20I.

II. M c G raw, Th. A., On intussusception of the vermiform appendix into the coecum. British medical Journal 1897. 9. Oktober.

12. H a a $\mathrm{l}$ er, Uber Darminvagination. Langenbecks Archiv f. klin. Chir. Bd. 68, Heft 3, Fall 9.

13. Jalaguier, Invaginations de l'appendice iléo-coecale. Bulletin der l'académie de médecine. Sitzung vom 7. November 1905. Referat: Zentralbl. f. Chir. Igo6.

14. M c Kidd, John. Edinburgh medical Journal 1858, I.

I5. K $\mathrm{riz}$, Ein Fall von ileocökaler Darminvagination. !Wiener med. Presse I896, Nr. 49 u. 50.

16. I. ed der hos e, Ein Fall von Appendicitis und ileocökaler Invagination. D. Zeitschr. f. Chir. 1915. Bd. 134, Heft 4 .

17. Lees, D. B. u. Quarry Silcock. Ileocoecal Intussusception; excision of involved intestine: recovery. British medical Journal 1898, p. 132.

18. M a r chand, Invaginatio ileocoecalis und Invaginatio iliaca. Ärtlicher Verein zu Marburg. Berlin. klin. Wochenschr. I896, Nr.6.

19. M a rx, Invaginatio ileocolica. Ärztlicher Verein zu Nürnberg. 18. April I90I. Münchn. med. Wochenschr. I901, 6. August.

20. $\mathrm{Ni}$ ederle, Dor Processus vermiformis als Ausgangspunkt der ileocökalen Invagination. Casopis lékar. ceskych 1904, p. 856. Referat: Zentralbl. f. Chir. I904.

21. Penrose u. Kellock, A case of intussusception of the coecum; reduction after laparotomy; recovery. The Lancet I898, 30. July.

22. Pitts, Seven cases of intussusception in infants treated by abdominal section with six recoveries. The Lancet 1897 , 12. June (case II).

23. Q u a dflieg, Uber einen Fall von chronischer ileocökaler Invagination. Münchn. med. Wochenschr. I901, Nr.27.

24. Ray, Four cases of acute ileocoecal intussusception, treatment by laparotomy with reduction or excision. The Lancet I900, 29. September.

25. Riede1, a) Der Mechanismus der Darmeinstülpung bei ëinem Kinde mit 3 Invaginationen, 2 descendierenden und einer ascendierenden. Mitteil. aus d. Grenzgebieten d. Med. u. Chir. Bd. I4, S. 218;

b) Eine seit 3 Jahren sich wiederholende, spontan zurückgehende, schließ. lich irreponible Invaginatio ileo-ileocolica. Mitteil. aus d. Grenzgebieten d. Med. u. Chir. Bd. r4, S. 229.

26. Sprenge l, Appendicitis. D. Chir. 1906. Lieferung 46 d. S. 453 u. 483.

27. Waterhouse, H., Intussusception of the vermiform Process. The Lancet I897, 20. November, p. I3I9. 
I94 Pollag, Giebt es eine primäre Invagination des Wurmfortsatzes usw.

28. Westermann, G. H., Weekblad van det Neederlandsch Tijdschrift vor Geneeskunde, No. 24, zit. u. refer. bei Ackermann.

29. Wilms, M., Der Ileus. D. Chir. 1906. Licferung $46 \mathrm{~g}$. S. $676 \mathrm{ff}$.

3o. Wright u. Knowles Renshaw, Chronic Intussusception of the appendix into the coecum; operation; recovery. British medical Journal I 897, June I2. 